\title{
Acute toxicity, cytotoxic, phytotoxic, muscle relaxant, analgesic, antispasmodic and antimicrobial potential of Cocculus pendulus
}

\author{
Muhammad Nafees, Sami Ullah*, Barkat Ullah and Muhammad Ibrar \\ Department of Botany, University of Peshawar, 25120-Pakistan \\ *Corresponding author's email: sami_jan69@yahoo.com \\ Citation \\ Muhammad Nafees, Sami Ullah, Barkat Ullah and Muhammad Ibrar. Acute toxicity, cytotoxic, phytotoxic, muscle \\ relaxant, analgesic, antispasmodic and antimicrobial potential of Cocculus pendulus. Pure and Applied Biology. \\ Vol. 8, Issue 2, pp1615-1630. http://dx.doi.org/10.19045/bspab.2019.80104
}

Received: $30 / 01 / 2019$ Revised: $29 / 05 / 2019$

Accepted: 03/05/2019

Online First: 15/05/2019

\section{Abstract}

The present experimental studies describes the acute toxic, cytotoxic, phytotoxic potential, analgesic, GIT motility and antimicrobial activity of Cocculus pendulus. The pharmacological profile has been evaluated by conducting acute toxic, cytotoxic, phytotoxic potential, analgesic, GIT motility and antimicrobial potentials. The plant is unsafe for consumption at higher doses, while safe at lower doses. The plant showed significant cytotoxic potential of Cocculus pendulus crude stem extract (CSE) with 7.82 LD50 value and Cocculus pendulus crude root extract (CRE) with 4.18 LD50 value. Phytotoxic potential was also significant (CSE) with 32.32 FI50 value and (CRE) with 24.68 FI50 value. Muscle relaxant effect was significant at $90 \mathrm{mg} / \mathrm{kg}$ both in CSE and CRE in traction and inclined tests which were 70\%, 85\%, 75\% and 80\% respectively. Analgesic activity were carried out via acetic acid induced writhing method while antispasmodic activity was done through charcoal motility method. $C$. pendulus stem extract (CSE), $C$. pendulus root extract $(\mathrm{CRE})$ and isolated fractions showed highly significant $(* * \mathrm{P}<0.01)$ analgesic effect at higher doses. CSE and CRE and isolated fractions at all doses reduced the motility of GIT in albino mice. CSE, CRE and their isolated fractions showed remarkable reduction in the zone amplitude against Proteus $s p$ and Xanthomonas $s p$. These effects of crude and isolated fractions justify its use in folkloric medicines for cytotoxic, herbicidal, pain relief and various gastro-intestinal disorders.

Keywords: Acute toxic; Analgesic; Antimicrobial; Antispasmodic; Cocculus pendulus; Cytotoxic; Muscle relaxant; Phytotoxic

\section{Introduction}

Cocculus pendulus Diels. Synonym: Cocculus leaeba DC. is locally known as "Parwatti". Its common names are Sag-elghorab (Arab), Pilwan (Rajasthani), Parwatti (Punjab) and Dusaratige (Telugu) [1]. This plant has been highly recorded from in subtropical and tropical zones in Pakistan, South Africa and India [2]. People in Pakistan and Afghanistan use the plant parts, especially the roots to cure fevers, including intermittent fever [1]. In Nigeria the root and leaves are used for this purpose while in Senegal the Toucouleur and Paul people use 
the bark of stem bark and root for the same purpose [3]. In Senegal the Toucouleur and Paul people use stem and root bark decoctions against intestinal parasites and gonorrhea [1]. The root has a great reputation in Senegal against biliousness and menstrual problems and as a diuretic [3]. It is also a part of medicines against jaundice, yellow fever, leprosy, syphilis and as an aphrodisiac in the area [4]. It has been recorded that many medicinal plants have been used for curing many diseases as traditional treatments for thousands of years throughout the world. In less developed countries especially in rural areas, the peoples use medicinal plants as primary source for treatment [5]. In developing countries about $80 \%$ of the people are dependent on herbal plants for their primary health care [6].

In the present study Cocculus pendulus has been examined against cytotoxic, phytotoxic, acute toxic, muscle relaxant, analgesic, antispasmodic and antimicrobial bioassays.

\section{Materials and methods}

\section{Collection and preservation of the plant}

The fresh stem and root of the experimental plant were collected in January 2015 from the Tribal area, Sama Bada Bera, Labi Khel, F.R Peshawar. Each specimen was cleaned and washed. Both stem and root were completely dried under the sun. Both specimens were grinded. After grinding the powdered drugs were kept in impermeable bottles and were used for different pharmacological bioassays.

\section{Preparation of extract}

Dried powder of stem and root of the plant were extracted with ethanol kept on rotary shaker for 48 hours. After that it was filtered, the filtrated solution were collected were processed in rotary to make the final volume $1 / 5$ of the original volume and stored in impermeable bottles at $4^{\circ} \mathrm{C}$ for further processing [7].

\section{Experimental animals}

Albino mice were used in all bioassays. Animals were purchased from National institute of health (NIH) Islamabad. The animals were provided with favorable laboratory conditions $\left(25^{\circ} \mathrm{C}\right)$ and were provided with standard food and water.

\section{Isolation of alkaloids fractions}

The methodology of [8] was adopted for the quantitative screening of total alkaloids. 100 $\mathrm{ml}$ of acetic acid $(10 \%)$ was taken in which $2 \mathrm{~g}$ crude ethanolic extract of stem and root were added. The solution was allowed to stand for 4 hours and then filtered. After filtration, the extracts were placed on a water bath for further concentration to reduce the volume to one-fourth. Precipitate formation occurred by the addition of Concentrated $\mathrm{NH} 4 \mathrm{OH}$ drop wise. Dilute $\mathrm{NH} 4 \mathrm{OH}$ was used for washing the collected precipitate. The obtained product was collected and dried.

\section{Isolation of flavonoids fraction}

Quantitative determination of flavonoids was carried out following the methodology of [9]. $2 \mathrm{~g}$ crude extract in $20 \mathrm{ml}$ hot distilled water of both root and stem were dissolved to make a solution in a beaker. The solution was then filtered and placed in refrigerator for 3 hours. $10 \mathrm{ml}$ ethyl acetate was added to the solution after refrigeration. The flavonoids become precipitated and were filtered. The filtrate was collected and dried.

\section{Acute toxic activity}

The stem (CSE) and root (CRE) crude ethanolic extract were subjected for the acute toxicity bioassay. Albino mice of either sex weighting $20-25$ grams were selected for bioassay. Seven groups of the animals were made. Each group was provided with 4 animals. Before performing the experiment, the animals were accustomed with the laboratory conditions. The control group were provided with normal saline and the remaining groups were provided the crude ethanolic extract of CSE (50, 70 and $90 \mathrm{mg} / \mathrm{kg}$ ) and CRE (50, 70 and $90 \mathrm{mg} / \mathrm{kg}$ ). The animals were continuously observed that either the extract show toxicity or safe for consumption [10]. 


\section{Cytotoxic bioassay}

The methodology of [11] was carried out to determine cytotoxic potential of the crude extracts. Cytotoxic bioassay has been conducted using Brine shrimp's lethality bioassay. Brine shrimp's eggs were kept in a container. The container were divided into two unequal parts contain $3.8 \%$ sea salt solution by a perforated septum. Brine shrimp's eggs were sprinkled in the smaller part of the container and was covered with black paper in order to create darkness, while the larger part of the tank was illuminated with electronic bulb. After two days of incubation, the eggs hatched and the nauplii start swimming towards the illuminated part of the container. $15 \mathrm{mg}$ crude ethanolic extract were dissolved in $1.5 \mathrm{ml}$ distilled water and stock solution were prepared. From the stock solution varying concentrations of 5,50 and $500 \mu \mathrm{g} / \mathrm{ml}$ were taken, equivalent to 10,100 and $1000 \mu \mathrm{g} / \mathrm{ml}$. 3 test tubes were taken for each concentration. Each test tube was provided with 10 brine shrimp larvae containing $5 \mathrm{ml}$ saline solution. After 24 hours the number of alive shrimp's were counted in each test tube with the help of magnifying glass. The percentage mortality data were calculated and the $50 \%$ lethal dose $\left(\mathrm{LD}_{50}\right)$ values were recorded [12].

\section{Phytotoxic activity}

The methodology of [11] was conducted for phytotoxic bioassay using Lemna minor for experiment. $20 \mathrm{mg}$ of the extract was dissolved in $2 \mathrm{ml}$ water from which different concentrations 10, 100, $1000 \mu \mathrm{g} / \mathrm{ml}$ were made. For each concentration 3 petri dishes were taken. Each petri dish was provided with $20 \mathrm{ml}$ E-medium. Other petri dishes (3 petri dishes for each) were supplied with Emedium taken as negative control and standard drug Atrazine taken as +ve control. 10 plants containing 3 fronds were kept under 12 hours day light in petri dishes. The fronds were observed regularly and their number were counted after three days. The following formula were used for recording the \% growth inhibition [10].

$$
\text { Inhibition } \%=\frac{(100-\text { Number of fronds in test sample })}{(\text { Number of fronds in negative control })} \times 100
$$

\section{Muscle relaxant activity Materials required}

Diazepam, distilled water, albino mice, rigid wire, inclined plane and selected parts crude extracts and isolated alkaloids and flavonoids fractions from the crude extracts.

\section{Traction test}

The experiment was conducted on a metal wire. The wire was fixed tightly in between two stands about $60 \mathrm{~cm}$ above the table. The animals were divided into control and test groups each with 4 animals. Group I was treated with normal saline (negative control) and group II was provided with diazepam (positive control) at $(10 \mathrm{ml} / \mathrm{kg})$ and $(1 \mathrm{mg} / \mathrm{kg})$ doses respectively. Rest of the groups were treated with CSE $(50,70$ and $90 \mathrm{mg} / \mathrm{kg}$ ) and CRE (50, 70 and 90mg/kg) respectively. All the animals were freely hanged by their hind legs after 30 minutes of the treatments and hanging time was recorded for 5 seconds. Hanging less than 5 seconds reflects the presence of muscle relaxant property otherwise absent [13].

\section{Inclined plane test}

The animals were divided into control and test groups each with 4 animals. Group I was treated with normal saline (negative control) and group II was provided with diazepam (positive control) at $(10 \mathrm{ml} / \mathrm{kg})$ and $(1 \mathrm{mg} / \mathrm{kg})$ doses respectively. Rest of the groups were treated with CSE $(50,70$ and $90 \mathrm{mg} / \mathrm{kg}$ ) and CRE (50, 70 and $90 \mathrm{mg} / \mathrm{kg}$ ) respectively. Each group was left on inclined screen and observed whether the effect of doses was significant to cause the mice to slide down of the screen or non-significant and the mice remained on the inclined slope [13]. 


\section{Analgesic bioassay}

\section{Acetic acid induced writhing test}

Analgesic activity was conducted on albino mice of either sex having 20-25 g weight. Animal were distributed into 20 groups $(\mathrm{n}=4)$. Diclofenac sodium $(10 \mathrm{mg} / \mathrm{kg})$ was given to Group-I and normal saline (10 $\mathrm{ml} / \mathrm{kg}$ ) were given to Group- II and other groups were treated with CSE (50, 70 and $90 \mathrm{mg} / \mathrm{kg}$ ) and its isolated Alkaloids from crude stem extract (ACSE) (15, 30 and
$45 \mathrm{mg} / \mathrm{kg}$ ) and Flavonoids from crude stem extract (FCSE) (15, 30 and $45 \mathrm{mg} / \mathrm{kg}$ ), and CRE $(50,70$ and $90 \mathrm{mg} / \mathrm{kg}$ ) and isolated Alkaloids from crude root extract (ACRE) $(15,30$ and $45 \mathrm{mg} / \mathrm{kg})$ and Flavonoids from crude root extract (FCRE) (15, 30 and $45 \mathrm{mg} / \mathrm{kg}$ ). After the above treatment acetic acid $(1 \%)$ were injected to the animals. After 5 min of acetic acid injection the writhing's were counted for 10 minutes $[14,15]$.

$$
\% \text { Analgesia }=100-\frac{\text { No. of writhing in tested animals }}{\text { No. of writhing in control animals }} \times 100
$$

\section{Antispasmodic activity}

\section{Procedure}

The methodology of [16] were carried out to conduct antispasmodic bioassay. Albino mice weighting 20-25g of either sex were used. Before commencing the experiment, Mice were deprived from food for 4 hours. Animals were divided into 20 groups. All the animals in each group were given $1 \mathrm{ml}$ charcoal meal orally. Immediately after administration the plant extracts CSE (50, 70 and $90 \mathrm{mg} / \mathrm{kg}$ ) and CRE $(50,70$ and $90 \mathrm{mg} / \mathrm{kg}$ ) and their isolated fractions from the crude extracts ACSE (15, 30 and $45 \mathrm{mg} / \mathrm{kg}$ ) and FCSE $(15,30$ and $45 \mathrm{mg} / \mathrm{kg})$ and ACRE (15, 30 and $45 \mathrm{mg} / \mathrm{kg}$ ) and FCRE $(15,30$ and $45 \mathrm{mg} / \mathrm{kg}$ ) respectively were injected. Each mouse was sacrificed by cervical dislocation after 30 minutes of charcoal meal test. In the intestine of each mice movement of charcoal was observed. Percentage inhibition of extracts was calculated.

\section{Statistical analysis}

SPSS- Software version-22 were used for data analysis by using one-way ANOVA, for multiple comparisons between control and test group. The probability level $\mathrm{P}<0.05$ was considered as significant while $\mathrm{P}<0.001$ as highly significant.

Antibacterial activity

Microorganisms used
4 pathogenic bacteria Staphylococcus aureus, Xanthomonas sp, Clavibacter and Proteus sp were used.

\section{Procedure}

The methodology of [17] was used, using agar disc diffusion method. Streptomycin was used as +ve control while blank discs were used as -ve control. 15 $\mu 1$ microbial suspension was spread on the nutrient agar plates. Whatman $(6 \mathrm{~mm}$ in diameter) were poured with $10 \mu \mathrm{l}$ of the CSE (100 and 1000ppm) and CRE (100 and 1000ppm) and their isolated fractions from the crude extracts ACSE (100 and 1000ppm) and FCSE (100 and 1000ppm) and ACRE (100 and 1000ppm) and FCRE (100 and $1000 \mathrm{ppm}$ ) solutions. Then, these plates were incubated for $24 \mathrm{~h}$ at $36^{\circ} \mathrm{C}$. After $24 \mathrm{hrs}$ zone inhibition was measured in comparison with the control Streptomycin a standard antibiotic.

\section{Results}

\section{Acute toxic activity}

C. pendulus stem and root were used to carry out their acute toxicity test. CSE $(100,150$ and $200 \mathrm{mg} / \mathrm{kg}$ ) and CRE $(100,150$ and $200 \mathrm{mg} / \mathrm{kg}$ ) showed $100 \%$ mortality, so the doses was diluted to CSE (80, 100 and $120 \mathrm{mg} / \mathrm{kg})$ and CRE (80, 100 and $120 \mathrm{mg} / \mathrm{kg}$ ), which showed $60 \%$ mortality. The doses were further diluted to CSE $(50,70$ and $90 \mathrm{mg} / \mathrm{kg}$ ) and $\mathrm{CRE}(50,70$ and $90 \mathrm{mg} / \mathrm{kg})$ which was safe and did not showed any 
mortality. So, these concentrations were said to be safe for consumption (Table 1).

Table 1. Acute toxic activity of $C$. pendulus

\begin{tabular}{|c|c|c|c|c|c|c|}
\hline Treatments & Dose mg/kg & $\begin{array}{c}\text { \% } \\
\text { Mortality }\end{array}$ & Dose $\mathbf{~ m g / k g}$ & $\begin{array}{c}\text { \% } \\
\text { Mortality }\end{array}$ & Dose mg/kg & $\begin{array}{c}\text { \% } \\
\text { Mortality }\end{array}$ \\
\hline \multirow{3}{*}{ CSE } & 100 & 100 & 80 & 0 & 50 & 0 \\
\cline { 2 - 7 } & 150 & 100 & 100 & 20 & 70 & 0 \\
\cline { 2 - 7 } & 200 & 100 & 120 & 40 & 90 & 0 \\
\hline \multirow{3}{*}{ CRE } & 100 & 100 & 80 & 0 & 50 & 0 \\
\cline { 2 - 7 } & 150 & 100 & 100 & 20 & 70 & 0 \\
\cline { 2 - 7 } & 200 & 100 & 120 & 40 & 90 & 0 \\
\hline
\end{tabular}

\section{Cytotoxic bioassay}

Brine shrimp's lethality bioassay of stem and root of $C$. pendulus crude ethanolic extract were evaluated. CSE doses 10, 100 and $1000 \mu \mathrm{g} / \mathrm{ml}$ showed $43.33 \%, 76.66 \%$ and
93.33\% mortality respectively with $\mathrm{LD}_{50}=7.82$ while CRE doses 10,100 and $1000 \mu \mathrm{g} / \mathrm{ml}$ showed $56.66 \%, 80 \%$ and $100 \%$ mortality with $\mathrm{LD}_{50}=4.18$ (Table 2, Fig. 1).

Table 2. Cytotoxic activity of C.pendulus stems and root on brine shrimps

\begin{tabular}{|c|c|c|c|c|c|}
\hline Treatments & Conc. $(\boldsymbol{\mu L})$ & $\begin{array}{c}\text { Total no. of } \\
\text { shrimp's }\end{array}$ & $\begin{array}{c}\text { No. of shrimps } \\
\text { survived }\end{array}$ & $\begin{array}{c}\text { \%age } \\
\text { inhibition }\end{array}$ & \multirow{2}{*}{ LD $_{\mathbf{5 0}}$} \\
\hline \multirow{3}{*}{ CSE } & 10 & 30 & 17 & 43.33 & \multirow{2}{*}{7.82} \\
\cline { 2 - 6 } & 100 & 30 & 7 & 76.66 & \multirow{2}{*}{4.18} \\
\cline { 2 - 6 } & 1000 & 30 & 2 & 93.33 & 56.66 \\
\hline \multirow{3}{*}{ CRE } & 10 & 30 & 13 & 80 & 100 \\
\cline { 2 - 6 } & 100 & 30 & 6 & 0 & \\
\cline { 2 - 6 }
\end{tabular}

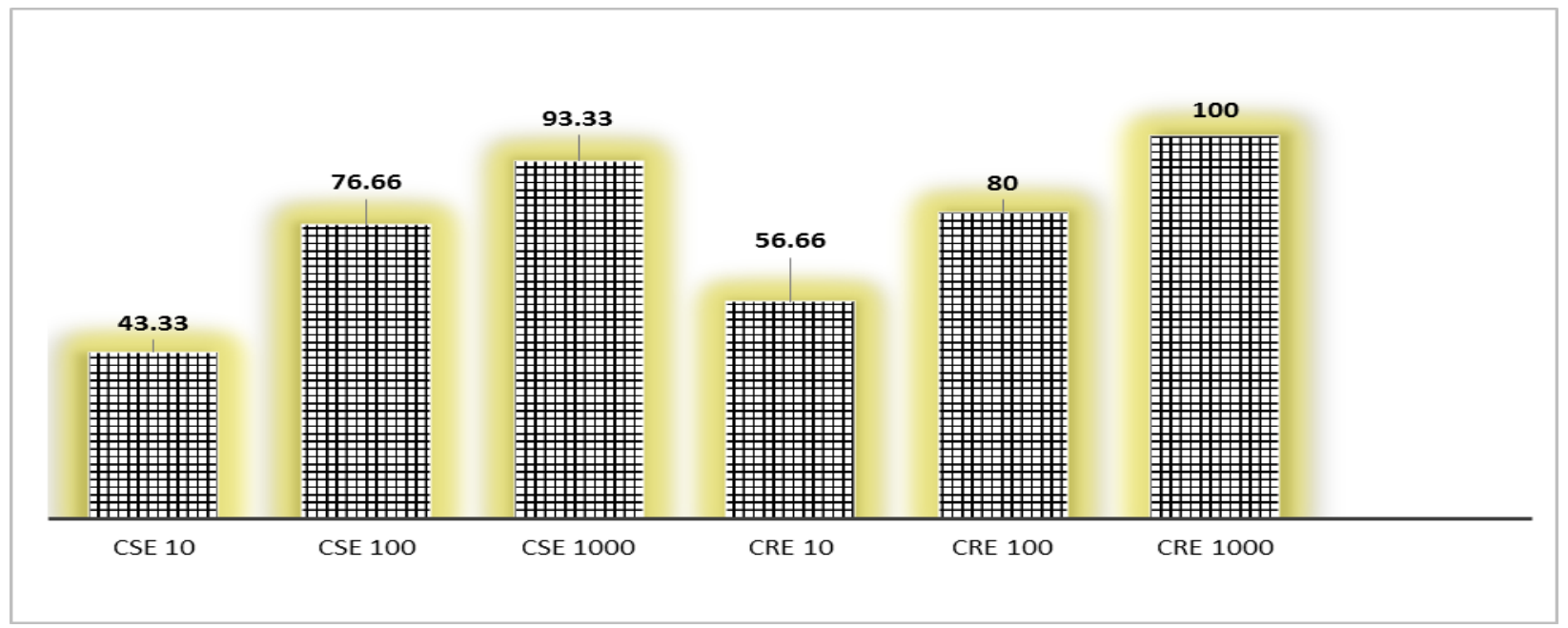

Figure 1. Percent cytotoxic effect of $C$ pendulus stems and root on brine shrimps

\section{Phytotoxic activity}

The stem and root of $C$. pendulus crude ethanolic extract were assessed using Lemna minor. CSE doses 10,100 and $1000 \mu \mathrm{g} / \mathrm{ml}$ showed $27.1 \%, 51 \%$ and $69 \%$ frond inhibition respectively with $\mathrm{FI}_{50}=32.32$, while CRE dose 10,100 and $1000 \mu \mathrm{g} / \mathrm{ml}$ showed $28.97 \%, 56.45 \%$ and $74.1 \%$ frond 
inhibition respectively with $\mathrm{FI}_{50}=24.68$ (Table 3, Fig. 2).

Table 3. Phytotoxic effect of $C$. pendulus stems and root on Lemna minor

\begin{tabular}{|c|c|c|c|c|c|}
\hline Parts & Conc. $(\mu \mathrm{l})$ & $\begin{array}{l}\text { No. of fronds } \\
\text { in test }\end{array}$ & $\begin{array}{l}\text { No. of fronds in ethanol } \\
\text { (-ve control) }\end{array}$ & $\begin{array}{c}\text { \%age } \\
\text { inhibition }\end{array}$ & $\mathrm{Fl}_{50}$ \\
\hline \multirow{3}{*}{ CSE } & 10 & 35 & \multirow{3}{*}{48} & 27.1 & \multirow{3}{*}{32.3} \\
\hline & 100 & 24 & & 51 & \\
\hline & 1000 & 12 & & 69 & \\
\hline \multirow{3}{*}{ CRE } & 10 & 35 & \multirow{3}{*}{48} & 28.97 & \multirow{3}{*}{24.68} \\
\hline & 100 & 22 & & 56.45 & \\
\hline & 1000 & 13 & & 74.1 & \\
\hline
\end{tabular}

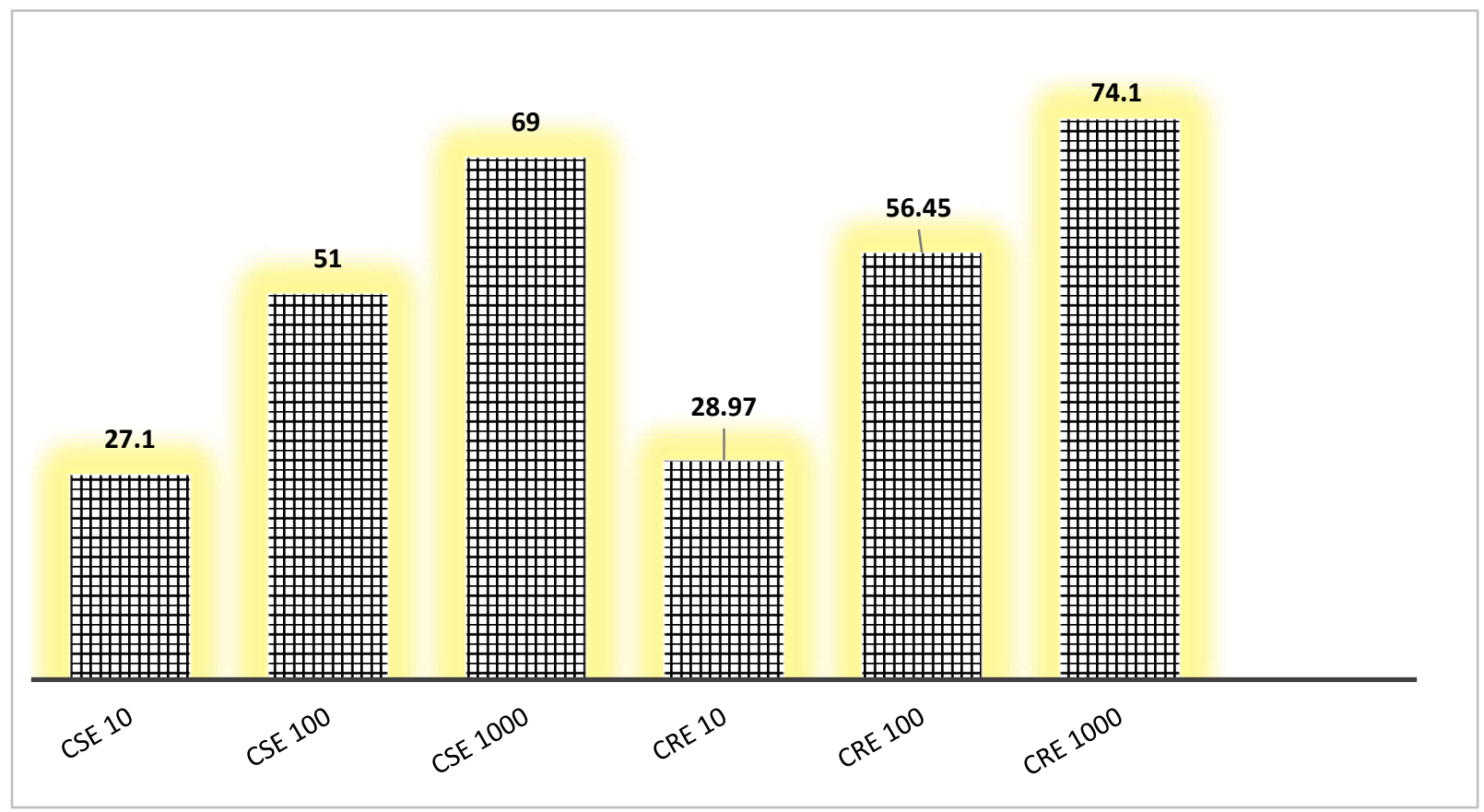

Figure 2. Percent phytotoxic effect of C. pendulus stem and root on Lemna minor

\section{Muscle relaxant bioassay Traction test}

C. pendulus stem and root were used to carry out muscle relaxant activity using traction test. CSE and CRE showed highest muscle relaxant activity at $90 \mathrm{mg} / \mathrm{kg}$ dose which were $75 \%$ and $80 \%$ recorded respectively (Table 4 , Fig. 3).

\section{Inclined plane test}

C. pendulus stem and root were used to carry out muscle relaxant activity using traction test. CSE and CRE showed highest muscle relaxant activity at $90 \mathrm{mg} / \mathrm{kg}$ dose $70 \%$ and $85 \%$ recorded respectively (Table 4, Fig. 3 ). 
Table 4. Percent muscle relaxant effect of $C$. pendulus

\begin{tabular}{|c|c|c|c|}
\hline \multirow{2}{*}{ Groups } & \multirow{2}{*}{ Dose } & Inclined plane test (\%) & Traction test (\%) \\
\cline { 3 - 4 } & $10 \mathrm{ml} / \mathrm{kg}$ & $\mathbf{3 0}$ minutes & $\mathbf{3 0}$ minutes \\
\hline Distilled water & $1 \mathrm{mg} / \mathrm{kg}$ & 100 & 100 \\
\hline \multirow{2}{*}{ Diazepam } & $50 \mathrm{mg} / \mathrm{kg}$ & 45 & 40 \\
\hline \multirow{3}{*}{ CSE } & $70 \mathrm{mg} / \mathrm{kg}$ & 60 & 65 \\
\cline { 2 - 4 } & $90 \mathrm{mg} / \mathrm{kg}$ & 70 & 75 \\
\hline \multirow{3}{*}{$\mathrm{CRE}$} & $50 \mathrm{mg} / \mathrm{kg}$ & 50 & 50 \\
\cline { 2 - 4 } & $70 \mathrm{mg} / \mathrm{kg}$ & 65 & 70 \\
\cline { 2 - 4 } & $90 \mathrm{mg} / \mathrm{kg}$ & 85 & 80 \\
\hline
\end{tabular}

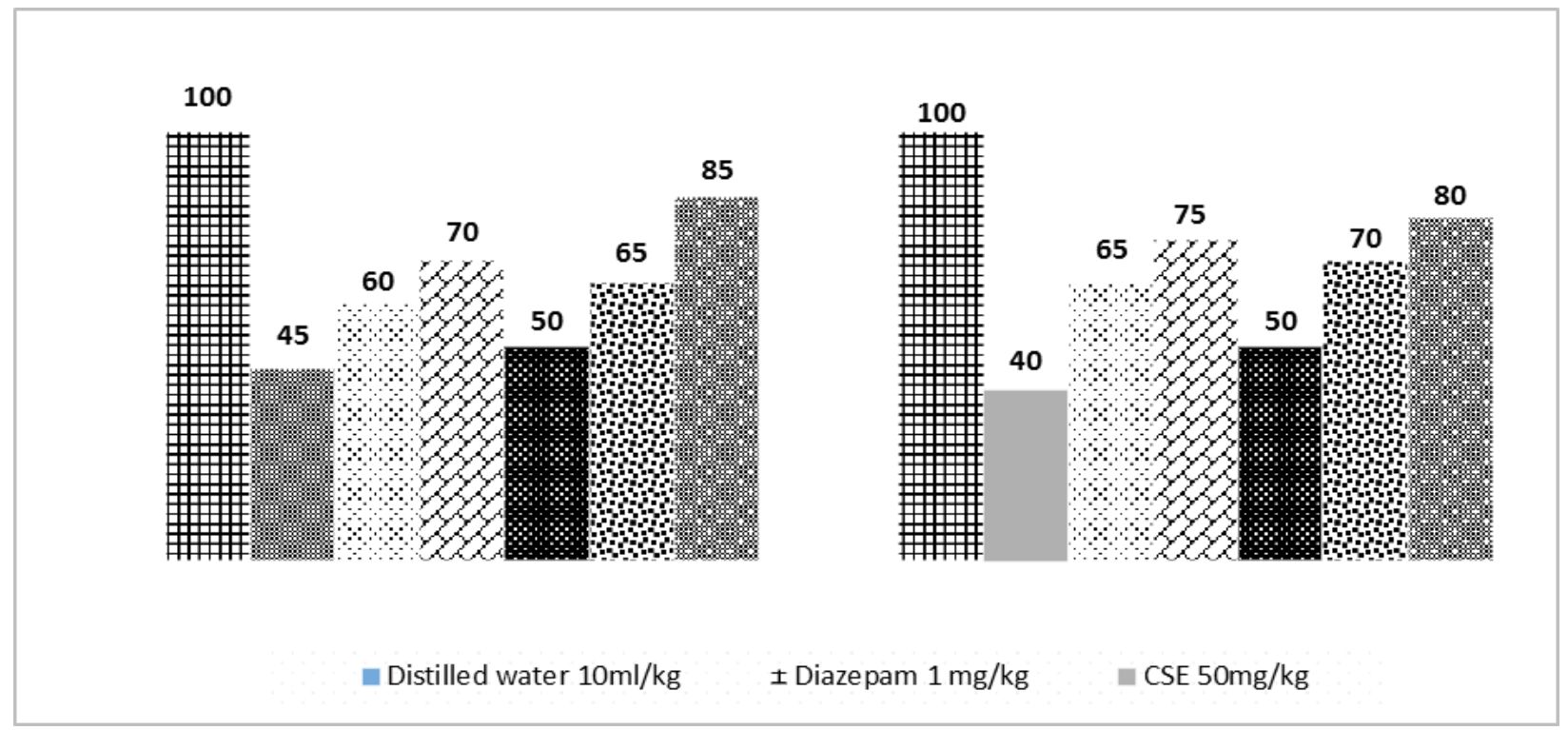

Figure 3. Percent muscle relaxant effect of $C$. pendulus

\section{Analgesic bioassay}

The crude ethanolic extract of stem and root and their isolated fractions of $C$. pendulus were assessed for analgesic activity by means of acetic-acid induced writhing model. CSE and CRE showed significant pain reduction at $(50,70$ and $90 \mathrm{mg} / \mathrm{kg})$ which were $(21.53$, 39.44 and $53.64 \%$ ) and (24.61, 43.07 and $53.84 \%$ ) respectively. CSE and CRE at 70 and $90 \mathrm{mg} / \mathrm{kg}$ doses showed highly significant
$(* * \mathrm{P}<0.01)$ results. Similarly, ACSE and ACRE at 30 and $45 \mathrm{mg} / \mathrm{kg}$ doses showed highly significant $(* * \mathrm{P}<0.01)$ results which were (38.01 and 55.23\%) and (49.23 and $58.46 \%$ ) respectively. FCSE and FCRE at 30 and $45 \mathrm{mg} / \mathrm{kg}$ doses showed highly significant $(* * \mathrm{P}<0.01)$ results which were $(32.73$ and $53.50 \%)$ and $(35.38$ and $55.38 \%)$ respectively (Table $5 \& 6$; Fig. $4 \& 5$ ). 
Table 5. Analgesic activity of $C$. pendulus stem

\begin{tabular}{|c|c|c|c|}
\hline \multirow{2}{*}{ Treatment } & Dose & No. of writhing's & \% writhing's \\
\hline Normal Saline & $10 \mathrm{ml} / \mathrm{kg}$ & $65.0 \pm 3.46$ & - \\
\hline Diclofenac sodium & $10 \mathrm{mg} / \mathrm{kg}$ & $18.0 \pm 1.14$ & 72.92 \\
\hline \multirow{3}{*}{ CSE } & $50 \mathrm{mg} / \mathrm{kg}$ & $51.0 \pm 1.53^{*}$ & 21.53 \\
\cline { 2 - 4 } & $70 \mathrm{mg} / \mathrm{kg}$ & $39 \pm 0.54^{* *}$ & 39.44 \\
\cline { 2 - 4 } & $90 \mathrm{mg} / \mathrm{kg}$ & $30 \pm 1.25^{* *}$ & 53.64 \\
\hline \multirow{3}{*}{ ACSE } & $15 \mathrm{mg} / \mathrm{kg}$ & $49.2 \pm 1.47^{*}$ & 24.61 \\
\cline { 2 - 4 } & $30 \mathrm{mg} / \mathrm{kg}$ & $40.2 \pm 1.62^{* *}$ & 38.01 \\
\cline { 2 - 4 } & $45 \mathrm{mg} / \mathrm{kg}$ & $29.1 \pm 2.35^{* *}$ & 55.23 \\
\hline \multirow{3}{*}{ FCSE } & $15 \mathrm{mg} / \mathrm{kg}$ & $54.22 \pm 2.83^{*}$ & 17.20 \\
\cline { 2 - 4 } & $30 \mathrm{mg} / \mathrm{kg}$ & $44.3 \pm 1.94^{* *}$ & 32.73 \\
\cline { 2 - 4 } & $45 \mathrm{mg} / \mathrm{kg}$ & $30.4 \pm 2.74^{* *}$ & 53.50 \\
\hline
\end{tabular}

All values are expressed as mean $\pm \mathrm{SD} * \mathrm{P}<0.05=$ Significant and $* * \mathrm{P}<0.01=$ Highly significant, compared to control

Table 6. Analgesic activity of $C$. pendulus root

\begin{tabular}{|c|c|c|c|}
\hline Treatment & Dose & No. of writhings & \% writhings \\
\hline Normal Saline & $10 \mathrm{ml} / \mathrm{kg}$ & $65 \pm 3.46$ & \\
\hline Diclofenac sodium & $10 \mathrm{mg} / \mathrm{kg}$ & $17.6 \pm 1.14$ & 72.92 \\
\hline \multirow{3}{*}{ CRE } & $50 \mathrm{mg} / \mathrm{kg}$ & $49.22 \pm 0.94^{*}$ & 24.61 \\
\cline { 2 - 4 } & $70 \mathrm{mg} / \mathrm{kg}$ & $37.71 \pm 1.33^{* *}$ & 43.07 \\
\cline { 2 - 4 } & $90 \mathrm{mg} / \mathrm{kg}$ & $30 \pm 0.55^{* *}$ & 53.84 \\
\hline \multirow{3}{*}{ ACRE } & $15 \mathrm{mg} / \mathrm{kg}$ & $46.33 \pm 0.45^{*}$ & 29.23 \\
\cline { 2 - 4 } & $30 \mathrm{mg} / \mathrm{kg}$ & $33.44 \pm 0.75^{* *}$ & 49.23 \\
\cline { 2 - 4 } & $45 \mathrm{mg} / \mathrm{kg}$ & $27.22 \pm 0.14^{* *}$ & 58.46 \\
\hline \multirow{3}{*}{ FCRE } & $15 \mathrm{mg} / \mathrm{kg}$ & $50 \pm 2.32^{*}$ & 23.07 \\
\cline { 2 - 4 } & $30 \mathrm{mg} / \mathrm{kg}$ & $42.22 \pm 1.34^{* *}$ & 35.38 \\
\cline { 2 - 4 } & $45 \mathrm{mg} / \mathrm{kg}$ & $29.43 \pm 0.94^{* *}$ & 55.38 \\
\hline
\end{tabular}

All values are expressed as mean $\pm \mathrm{SD} * \mathrm{P}<0.05=$ Significant and $* * \mathrm{P}<0.01=$ highly significant, compared to control

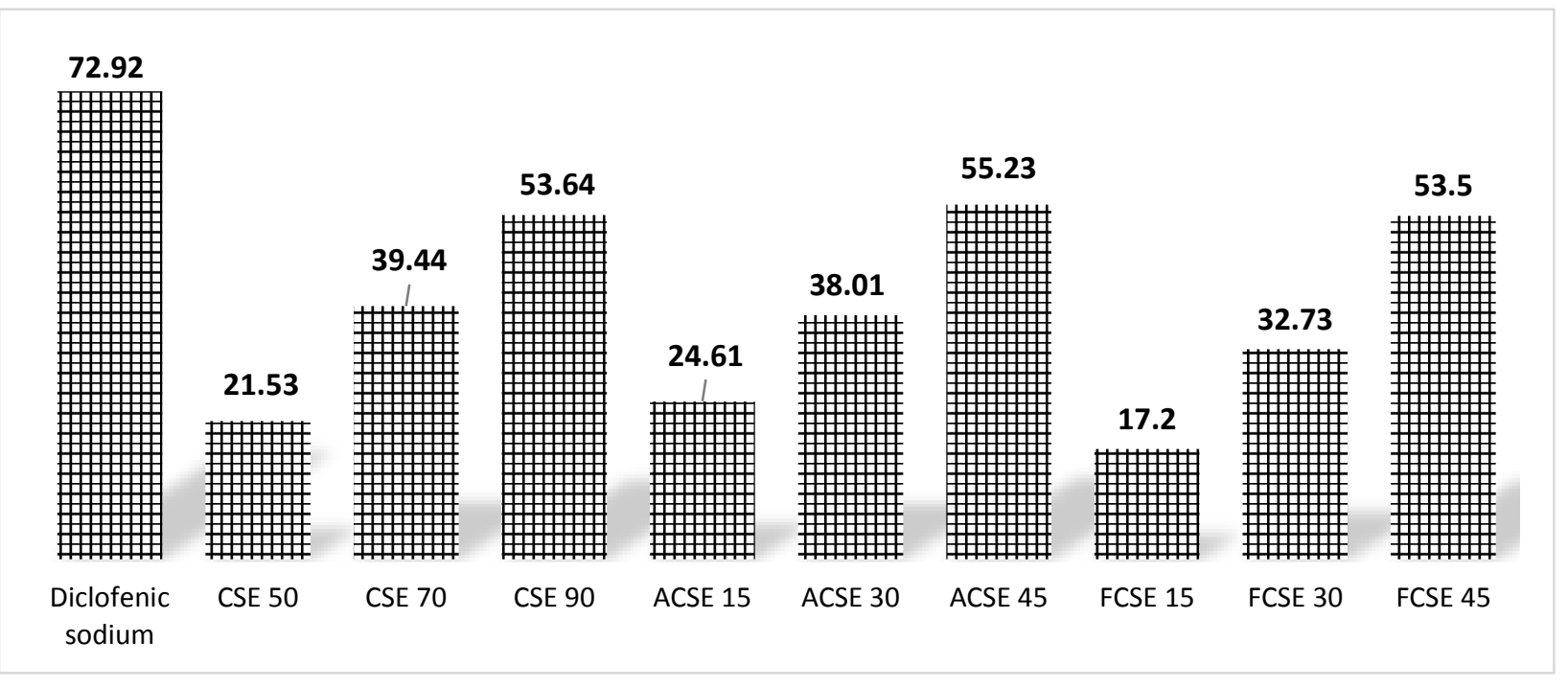

Figure 4. Percent analgesic activity of $C$. pendulus stem 


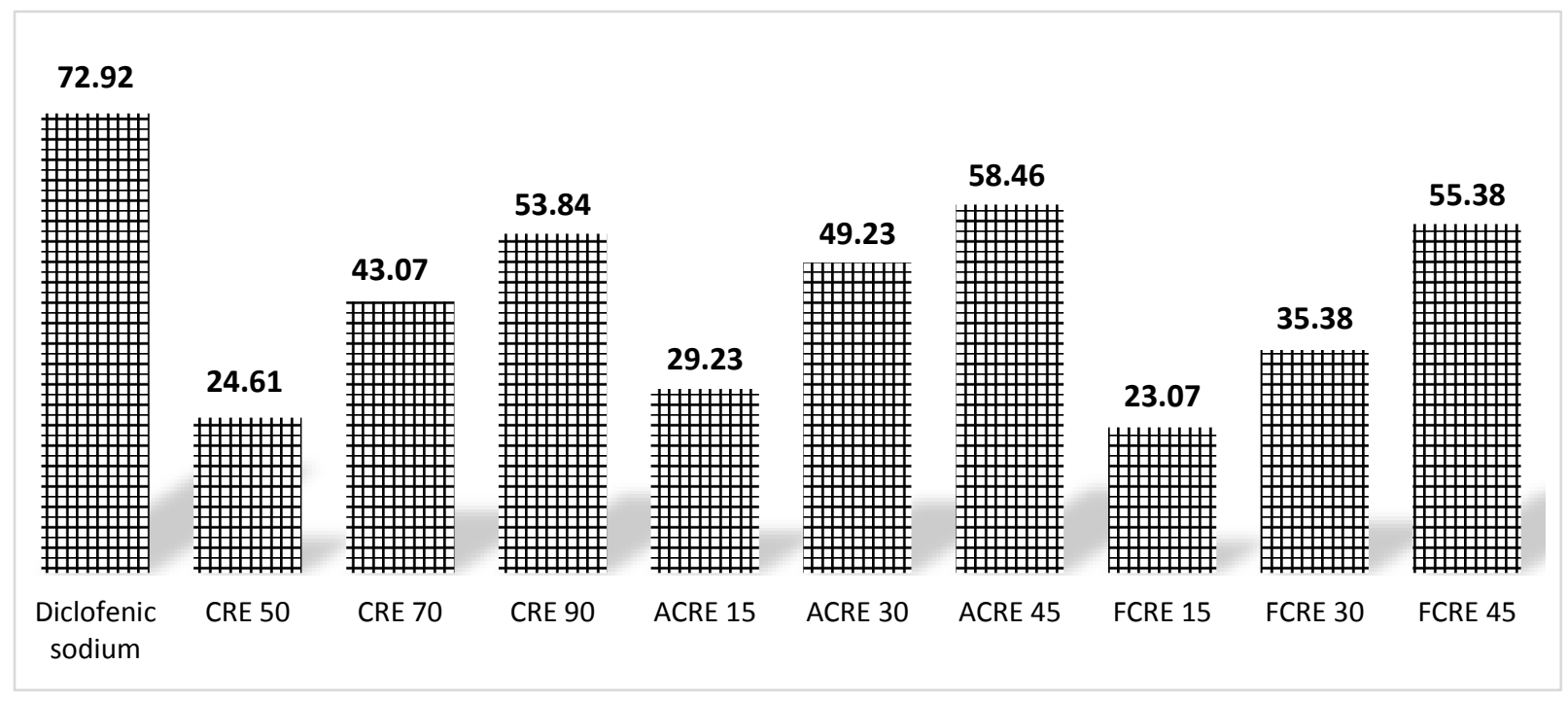

Figure 5. Percent analgesic activity of $C$. pendulus root

\section{Antispasmodic bioassay}

The stem and root crude ethanolic extract and their isolated fractions of $C$. pendulus were evaluated for antispasmodic bioassay using charcoal meal test. CSE and CRE at all doses $(50,70$ and $90 \mathrm{mg} / \mathrm{kg})$ showed highly significant $(* * \mathrm{P}<0.01)$ results which were (70.37, 74.46 and $81.79 \%)$ and $(80.83,84.49$ and $85.96 \%$ ) charcoal movement in intestine respectively. Similarly, ACSE and ACRE at all doses $(15,30$ and $45 \mathrm{mg} / \mathrm{kg})$ showed highly significant $(* * \mathrm{P}<0.01)$ results which were $(71.52,77.36$ and $81.25 \%)$ and $(78.10$, 82.87 and $85.59 \%$ ) charcoal movement in intestine respectively. FCSE and FCRE also showed highly significant $(* * \mathrm{P}<0.01)$ results at all doses $(15,30$ and $45 \mathrm{mg} / \mathrm{kg})$ which were $(66.15,71.17$ and $79.70 \%)$ and $(75.16,79.06$ and $84.90 \%$ ) charcoal movement in intestine respectively (Table $7 \& 8$; Fig. $6,7 \& 8$ ).

Table 7. Antispasmodic activity of of $C$. pendulus stem

\begin{tabular}{|c|c|c|c|c|}
\hline Treatment & Dose & $\begin{array}{c}\text { Total length of } \\
\text { intestine }(\mathbf{c m})\end{array}$ & $\begin{array}{c}\text { Distance covered by } \\
\text { charcoal }(\mathbf{c m})\end{array}$ & $\begin{array}{c}\text { \% distance covered } \\
\text { by charcoal }\end{array}$ \\
\hline Control & $10 \mathrm{ml} / \mathrm{kg}$ & $59.33 \pm 0.07$ & $15.17 \pm 0.27$ & 74.43 \\
\hline Castor oil & $10 \mathrm{ml} / \mathrm{kg}$ & $55.75 \pm 0.10$ & $33.25 \pm 0.17$ & 40.35 \\
\hline \multirow{4}{*}{ CSE } & $50 \mathrm{mg} / \mathrm{kg}$ & $51.57 \pm 0.68$ & $15.28 \pm 1.34^{* *}$ & 70.37 \\
\cline { 2 - 5 } & $70 \mathrm{mg} / \mathrm{kg}$ & $48.33 \pm 1.15$ & $12.34 \pm 0.13^{* *}$ & 74.46 \\
\cline { 2 - 5 } & $90 \mathrm{mg} / \mathrm{kg}$ & $49.44 \pm 1.02$ & $9.023 \pm 2.32^{* *}$ & 81.79 \\
\hline \multirow{3}{*}{ ACSE } & $15 \mathrm{mg} / \mathrm{kg}$ & $50.33 \pm 0.57$ & $14.13 \pm 0.97^{* *}$ & 71.52 \\
\cline { 2 - 5 } & $30 \mathrm{mg} / \mathrm{kg}$ & $49.21 \pm 0.59$ & $11.14 \pm 0.43^{* *}$ & 77.36 \\
\cline { 2 - 5 } & $45 \mathrm{mg} / \mathrm{kg}$ & $48.66 \pm 1.52$ & $9.12 \pm 1.33^{* *}$ & 81.25 \\
\hline \multirow{3}{*}{$\mathrm{FCSE}$} & $15 \mathrm{mg} / \mathrm{kg}$ & $51.23 \pm 0.54$ & $17.34 \pm 0.98^{* *}$ & 66.15 \\
\cline { 2 - 5 } & $30 \mathrm{mg} / \mathrm{kg}$ & $49.34 \pm 1.23$ & $14.22 \pm 1.23^{* *}$ & 71.17 \\
\cline { 2 - 5 } & $45 \mathrm{mg} / \mathrm{kg}$ & $49.87 \pm 2.13$ & $10.12 \pm 1.54^{* *}$ & 79.70 \\
\hline
\end{tabular}

All values are expressed as mean $\pm \mathrm{SD} * * \mathrm{P}<0.01=$ Highly significant, compared to control 
Table 8. Antispasmodic activity of $C$. pendulus root

\begin{tabular}{|c|c|c|c|c|}
\hline Treatment & Dose & $\begin{array}{c}\text { Total length of } \\
\text { intestine }(\mathbf{c m})\end{array}$ & $\begin{array}{c}\text { Distance covered } \\
\text { by charcoal (cm) }\end{array}$ & $\begin{array}{c}\text { \% distance covered } \\
\text { by charcoal }\end{array}$ \\
\hline Control & $10 \mathrm{ml} / \mathrm{kg}$ & $59.33 \pm 0.07$ & $15.17 \pm 0.27$ & 74.43 \\
\hline \multirow{2}{*}{ Castor oil } & $10 \mathrm{ml} / \mathrm{kg}$ & $55.75 \pm 0.10$ & $33.25 \pm 0.17$ & 40.35 \\
\hline \multirow{3}{*}{ CRE } & $50 \mathrm{mg} / \mathrm{kg}$ & $53.54 \pm 1.32$ & $10.26 \pm 1.64 * *$ & 80.83 \\
\cline { 2 - 5 } & $70 \mathrm{mg} / \mathrm{kg}$ & $52.23 \pm 0.21$ & $8.10 \pm 0.24 * *$ & 84.49 \\
\cline { 2 - 5 } & $90 \mathrm{mg} / \mathrm{kg}$ & $49.87 \pm 1.65$ & $7 \pm 0.43^{* *}$ & 85.96 \\
\hline \multirow{3}{*}{ ACRE } & $15 \mathrm{mg} / \mathrm{kg}$ & $50.37 \pm 0.97$ & $11.03 \pm 0.52^{* *}$ & 78.10 \\
\cline { 2 - 5 } & $30 \mathrm{mg} / \mathrm{kg}$ & $50.10 \pm 1.32$ & $8.58 \pm 1.23 * *$ & 82.87 \\
\cline { 2 - 5 } & $45 \mathrm{mg} / \mathrm{kg}$ & $49.43 \pm 0.54$ & $7.12 \pm 0.21 * *$ & 85.59 \\
\hline \multirow{3}{*}{ FCRE } & $15 \mathrm{mg} / \mathrm{kg}$ & $56.25 \pm 0.65$ & $13.97 \pm 2.1 * *$ & 75.16 \\
\cline { 2 - 5 } & $30 \mathrm{mg} / \mathrm{kg}$ & $54.37 \pm 0.76$ & $11.38 \pm 1.62 * *$ & 79.06 \\
\cline { 2 - 5 } & $45 \mathrm{mg} / \mathrm{kg}$ & $55.73 \pm 1.74$ & $8.41 \pm 1.37 * *$ & 84.90 \\
\hline
\end{tabular}

All values are expressed as mean $\pm \mathrm{SD} * * \mathrm{P}<0.01=$ Highly significant, compared to control

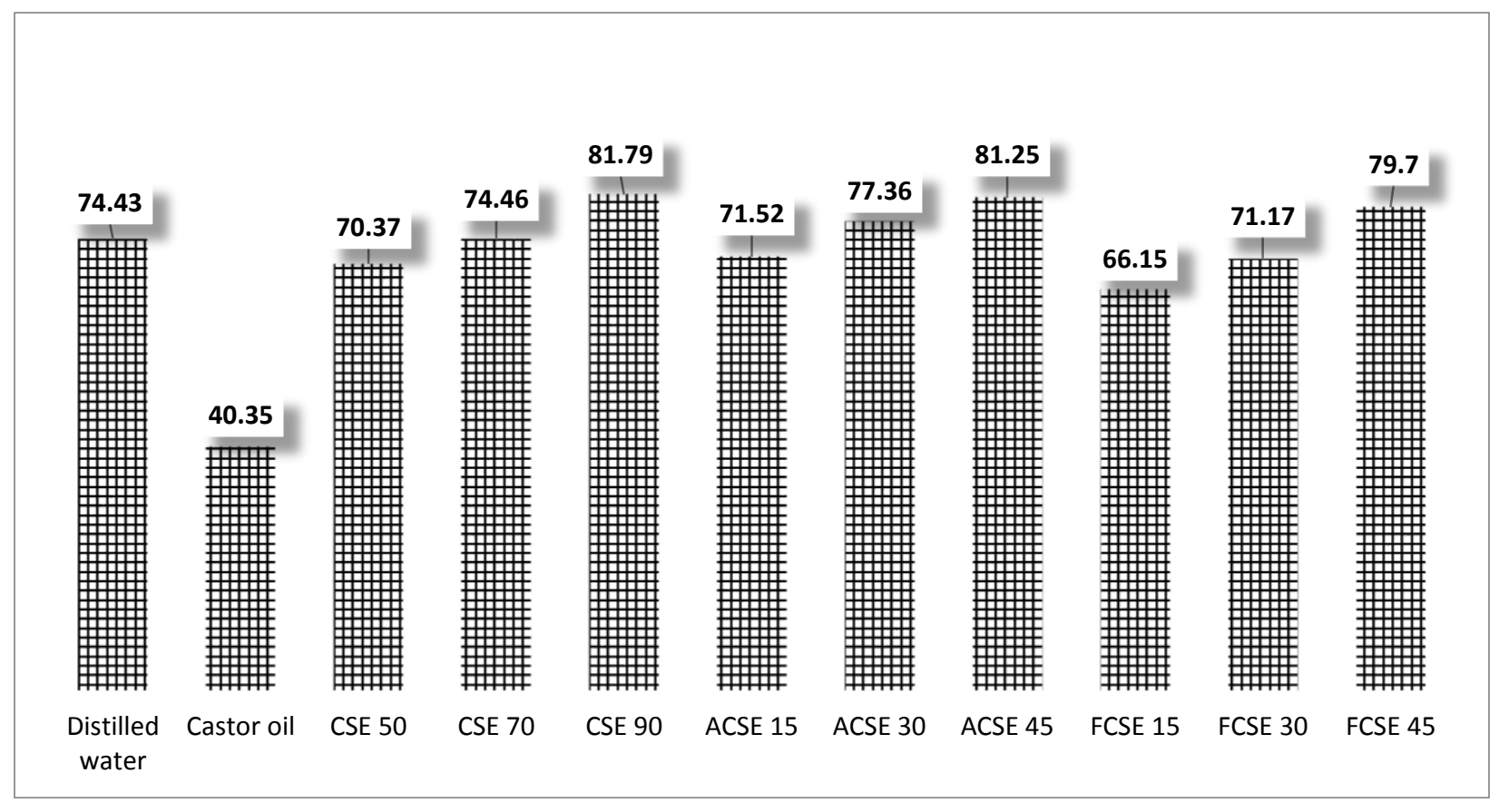

Figure 6. Percent antispasmodic activity of $C$. pendulus stem 


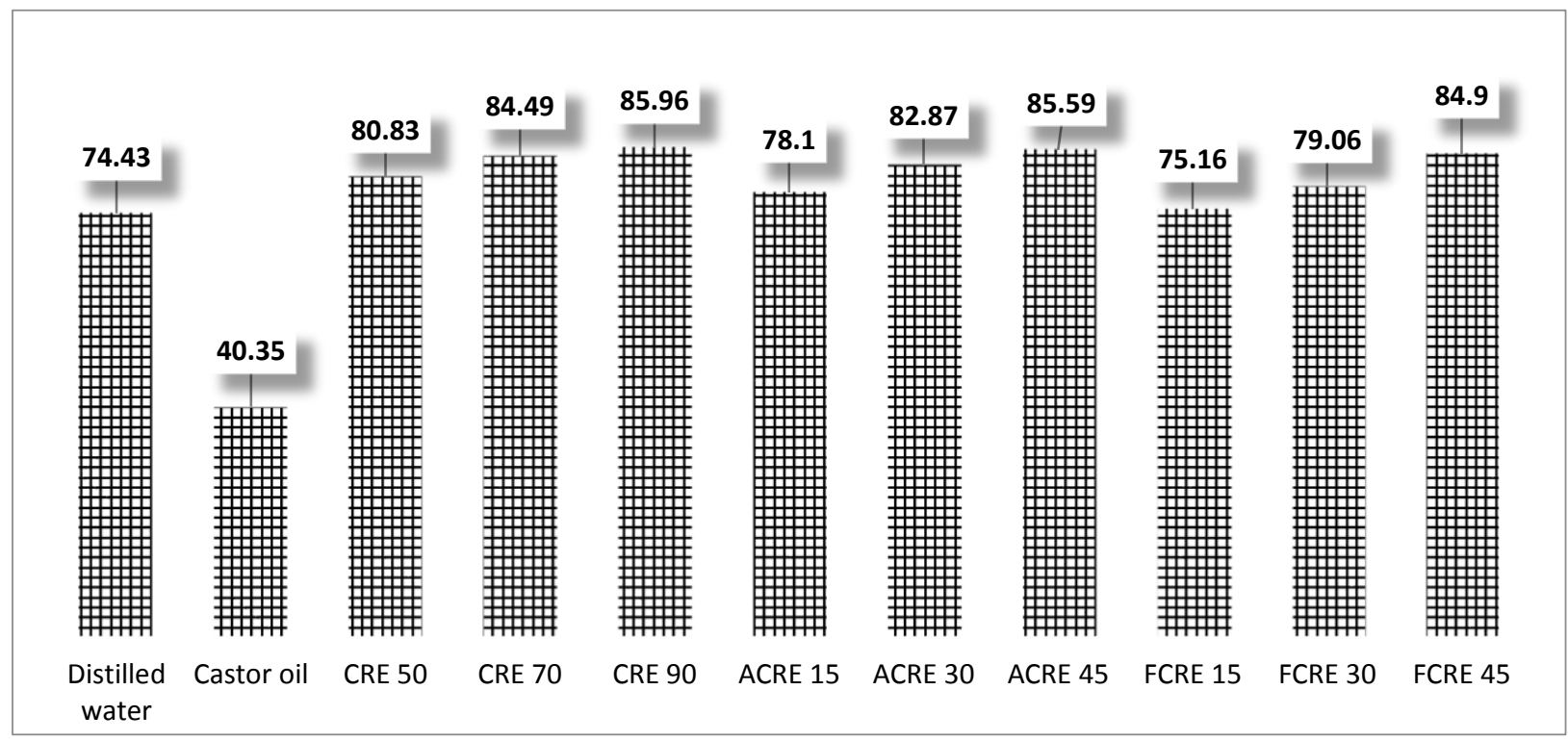

Figure 7. Percent antispasmodic activity of of $C$. pendulus root

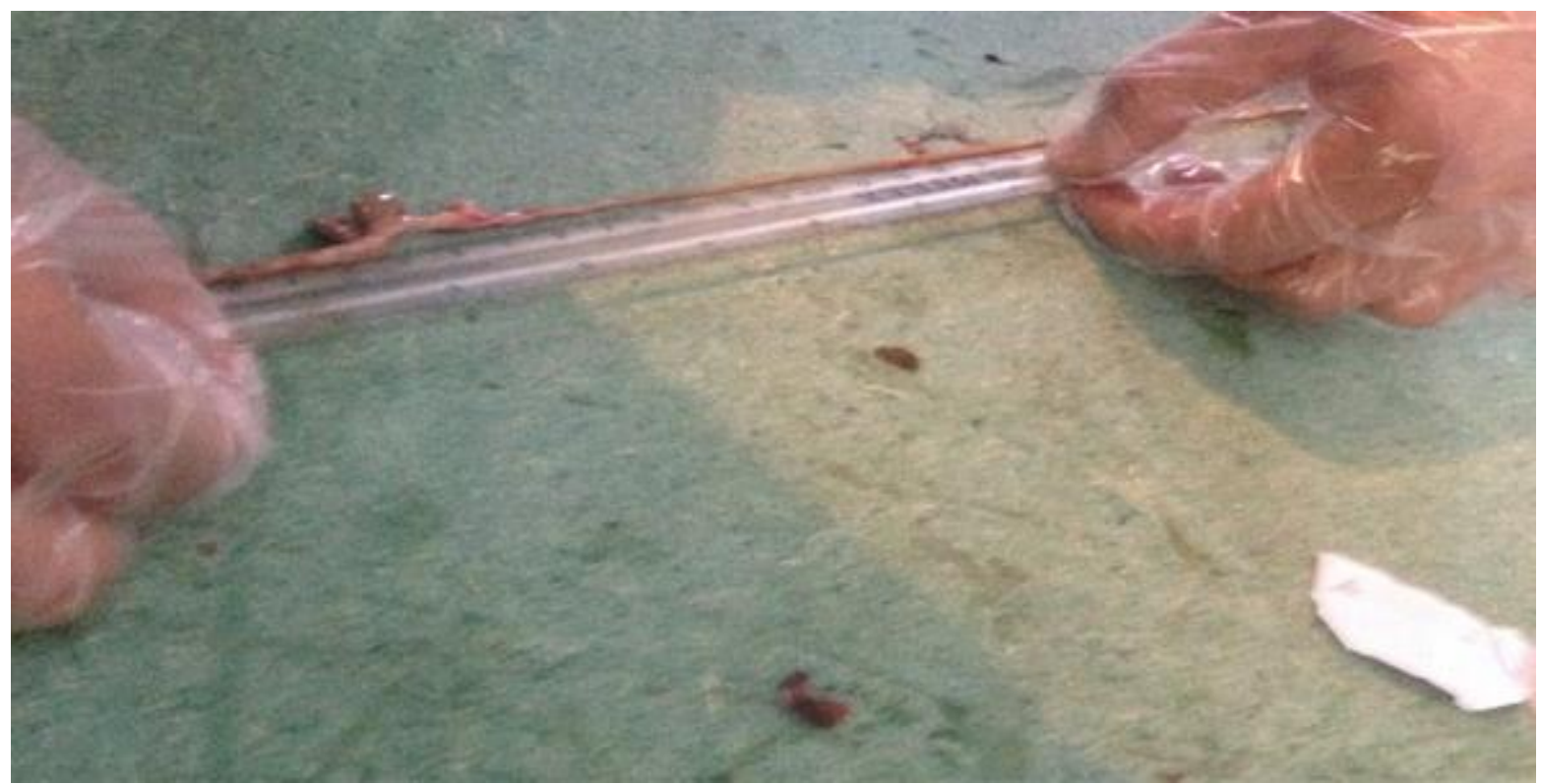

Figure 8. Measuring charcoal movement in mice intestine

\section{Antibacterial bioassay}

The stem and root crude ethanolic extracts and their isolated fractions of $C$. pendulus were assessed using agar disc diffusion method for antimicrobial activity. The stem and its isolated fractions at all doses showed remarkable results. The highest zone amplitude reduction was noted against Xanthomonas sp $(17 \mathrm{~mm})$ by CSE, while
ACSE produced (18mm) against Xanthomonas $s p$ and FCSE produced (18mm) against Proteus sp. Similarly, the root and its isolated fractions also showed remarkable zones of inhibition at higher doses. The highest zone amplitude reduction (18mm) was noted against Proteus $s p$, while ACRE produced $(18 \mathrm{~mm})$ zone of inhibition against Proteus $s p$ and FCRE produced 
(18mm) against Xanthomonas sp (Table 9;

Fig. 9).

Table 9. Antibacterial activity of $C$. pendulus stem and root

\begin{tabular}{|c|c|c|c|c|c|c|}
\hline \multirow{2}{*}{$\begin{array}{c}\text { S. } \\
\text { No. }\end{array}$} & \multirow{2}{*}{ Treatments } & \multirow{2}{*}{ Dose } & S. aureus & Clavibacter & Proteus sp & Xanthomonas sp \\
\hline & & & \multicolumn{4}{|c|}{ Zone of inhibition (mm) } \\
\hline 1 & Streptomycin & $0.5 \mathrm{mg} / \mathrm{ml}$ & 18 & 20 & 21 & 19 \\
\hline \multirow{2}{*}{2} & \multirow{2}{*}{ CSE } & 100ppm & 9 & 9 & 7 & 9 \\
\hline & & $1000 \mathrm{ppm}$ & 15 & 16 & 15 & 17 \\
\hline \multirow{2}{*}{3} & \multirow{2}{*}{ CRE } & $100 \mathrm{ppm}$ & 10 & 9 & 10 & 9 \\
\hline & & $1000 \mathrm{ppm}$ & 17 & 18 & 18 & 16 \\
\hline \multirow{2}{*}{4} & \multirow{2}{*}{ ACSE } & 100ppm & 11 & 10 & 9 & 10 \\
\hline & & $1000 \mathrm{ppm}$ & 17 & 17 & 18 & 18 \\
\hline \multirow{2}{*}{5} & \multirow{2}{*}{ FCSE } & $100 \mathrm{ppm}$ & 10 & 9 & 10 & 8 \\
\hline & & $1000 \mathrm{ppm}$ & 16 & 17 & 18 & 17 \\
\hline \multirow{2}{*}{6} & \multirow{2}{*}{ ACRE } & 100ppm & 10 & 9 & 8 & 9 \\
\hline & & $1000 \mathrm{ppm}$ & 17 & 18 & 18 & 17 \\
\hline \multirow{2}{*}{7} & \multirow{2}{*}{ FCRE } & $100 \mathrm{ppm}$ & 10 & 11 & 9 & 11 \\
\hline & & 1000ppm & 18 & 17 & 18 & 18 \\
\hline
\end{tabular}

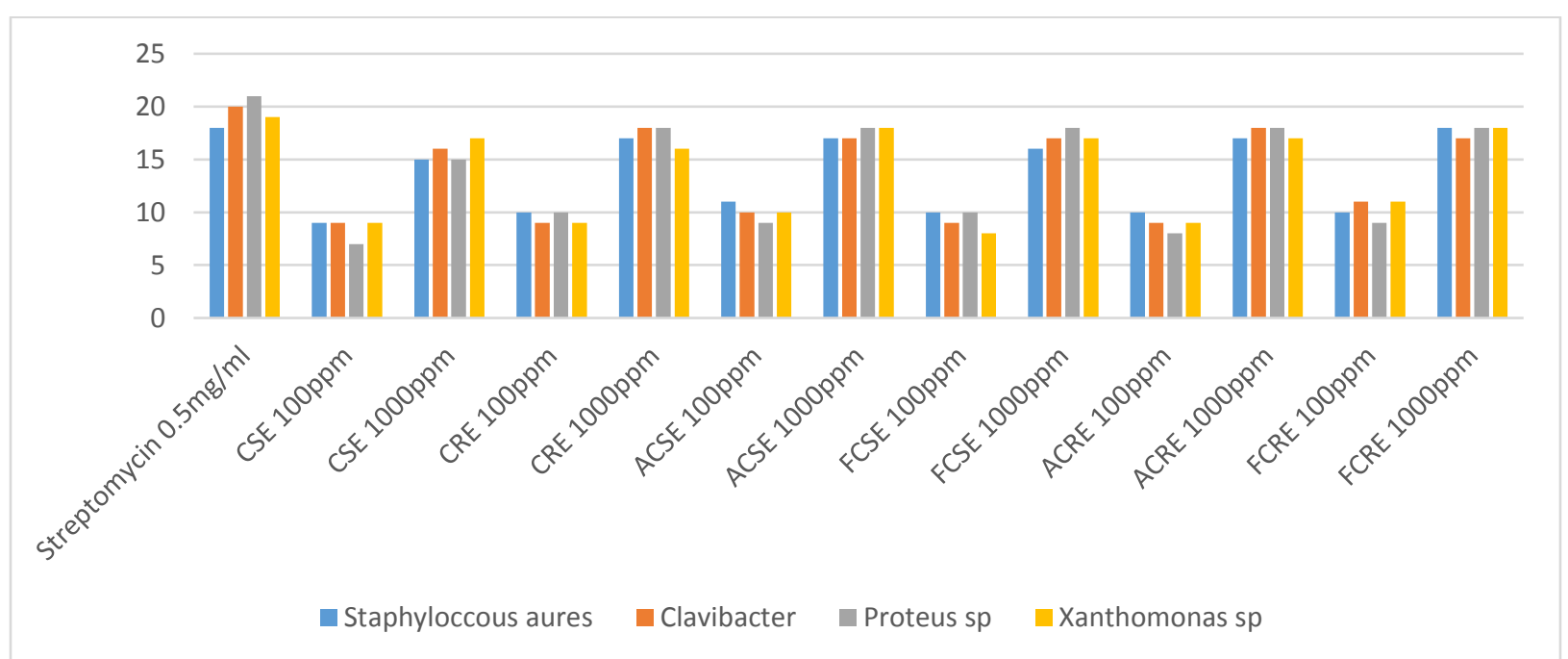

Figure 9. Antibacterial activity of $C$. pendulus stem and root

\section{Discussion}

The above results of acute toxic bioassay revealed that this plant is not safe for the consumption at higher doses, while safe at lower doses as discussed above. Brine shrimp cytotoxic bioassay a simple, economical and efficient method of screening of test articles for anti-cancer potential. When CSE and CRE were tested against anti-cancer potential

both the extracts showed significant anticancer potential. This indicate that this plant is good for the therapy of cancer. Weeds interference noticeably responsible for the huge economic loss to the quality and quantity of agricultural crops all over the 
world. It is estimated in US that weeds cause a loss of at least $12 \%$ costing to nearly US\$ 33 billion while the situation is more alarming in developing countries [18]. As an agriculture country, Pakistan yielding high quality and quantity of various cereals and crops. Large quantity of these crops may be damaged due to poor weed control strategies. In agricultural sectors, synthetic herbicides are widely used for the weed control. However, various factors such as water and soil pollution, herbicide residues, detrimental effects and herbicide-resistant weed populations restricted the use of synthetic herbicides. The degree of harm caused by insects and diseases are less than that cause by uncontrolled weeds, but its effects are unseen. Due to competition for sunlight, water and fertilizer, they also reduce the crops yield. Furthermore, weeds are actively involved in habitat provision for insects which help in spreading of disease. So, for increasing the production of various crops, weeds controlling is very essential. In our present study both the extracts showed significant phytotoxic potential, which indicate that this plant has good weedicidal potential [18].

Traction and inclined plane tests are techniques use for the determination of muscle relaxant properties in animals. The animals in these models can spend time on wire and inclined plane, less time spent more indicates a muscle relaxant effect of a tested material. Our findings reveal that crude ethanolic extract of both stem and root exhibit significant activity. So, it is clearly indicated the muscle relaxant active substances (secondary metabolites) of the plant are concentrated in these two extracts. In addition, our results are well in lineage with the standard drug diazepam used in the study

Analgesic potential of medicinal plants can be determined by using Acetic acid-induced writhing method [19]. In acetic acid model pain sensation is caused by the production of inflammation causing factors arachidonic acid from phospholipids tissues through an enzyme known as cyclo-oxygenase (COX), that release prostaglandin specifically PGE2 and PGF $2 \alpha$. In peritoneal fluids, the level of lipoxygenase also become up which increases capillary permeability [20]. The substance preventing the writhing by reducing pain due to inhibiting prostaglandin synthesis, this is peripheral analgesic effect [19]. In the current experiment, the results of crude extract and fractions showed remarkable reduction of writhing reflux. These observations strongly suggested that $C$. pendulus and its fractions has good analgesic potential.

Various other investigators like [3, 21-27] carried out similar activity on several medicinal plant from various families like Hyptis suaveolens, Artocarpus lakoocha, Aphanamixis polystachya, Kalanchoe pinnata, Justicia prostrata, Catharanthus roseus and Skimmea. Laureola reported a good significant activity of these plants on mice and various other test using different test and methods. The workers suggested that the analgesic effect of these is due the presence of secondary phytochemical especially flavonoids and alkaloids use for analgesia. Hence, these all workers strongly supported and in line with our research because in $C$. pendulas the alkaloids and flavonoids good effect as compared to crude extracts, hence it is suggested that the specific alkaloids and flavonoids responsible for treatment of analgesia should be determined, characterized, isolated and should be used instead of haphazard synthetic and expansive drugs as natural, economic, easily available and safe source medicines.

Diarrhea is a very common ailment and drastic disease in most of the tropical countries of the world causing millions of deaths every year [28]. Along with modern advance synthetic drugs the herbal medicines 
are mostly effective and economic therapy for diarrhea in several Asian countries. Numerous medicinal plants have been testified to be most effective in safe treatment of diarrhea dysentery with no side effect. $C$. pendulus stem and root crude extract and isolated fractions showed significant results against diarrhea. In folk medicines, for treatment of gastrointestinal ailments many plants can be used. Nowadays many people also turn to the use of natural drugs for the treatment of intestinal complications. Several other researchers also agree with us as [29. 32] reported significant antispasmodic activities various plants like Swertia chirata, Myrtus communis. Manilkara zapota, Cynanchum viminale, Symplocos paniculata, and Withania somnifera in gastrointestinal motility and castor oil induced diarrhea in mice and hence suggested study on medicinal plants provides basis for the vernacular use in gastrointestinal system. We also suggested the $C$. pendulus as a good safe and natural antispasmodic plant because it shows a significant reduction in custard oil induced diarrhea in mice and suggested it further be advance explored and specific responsible substances must be isolated.

Plants extracts are important source of chemotherapeutic and antibiotic agents' due to existence of combination of primary and secondary active and non-active metabolites. The Unani System of Medicine still need to testify plants and their crude extracts according to the recent measures to confirm their active potential. Many reports showed that plant crude drugs with no side effect and reaction have good antibacterial, antifungal and anti-inflammatory potential [33]. Plants chemical are natural antimicrobial agents are considered to be environment friendly used as bio-control agents and easily available [34]. Various pure secondary metabolites and active constituents have been identified, isolated and used as effective natural drug for treatment of dangerous human diseases. The result obtained from present investigation concluded that the crude extract and isolated fractions showed significant results against all bacterial strains. Similar antimicrobial bioassay was conducted by [35] on Bligha sapida against five bacteria and five fungal and reported significant growth inhibitory activity. [36] Used methanolic extracts and various fractions of Cardiospermum halicacabumon and reported that ethanolic extract showed good results. [37] Worked on Impatiens bicolor [38] used Nephelium lappaceum against pathogenic bacteria and reported similar dose dependent zones of inhibition against bacteria. [39] Proved that Litchi leaf contained luteoline, epicatchin, procyanidin and rutin which are strongest antimicrobial against. All these studies strongly supported our work. Hence it is suggested that $C$. pendulus may also be used as antimicrobial drug. Therefore, this has prominent perspectives in developing antimicrobial agents through further biological research.

\section{Conclusion}

In conclusion, we can say that Cocculus pendulus contain active ingredients possessing cytotoxic, phytotoxic, muscle relaxant, analgesic, antispasmodic and antimicrobial potential. Our present findings provide a way for the exploration of the plant and isolation of the active constituents for further advance studies.

\section{Authors' contributions}

Conceived and designed the experiments: B Ullah, Performed the experiments: M Nafees, Analyzed the data: S Ullah, Contributed materials/ analysis/ tools: M Ibrar, Wrote the paper: M Nafees.

\section{References}

1. Chopra RN, Chopra IC, Handa KL \& Kapoor LD (1958). UN Dhar and Sons Pvt. Ltd; Calcutta, pp 58.

2. Siddique MA (1974). Menispermaceae; Flora of Pakistan, Department of Botany; University of Karachi, Karachi, pp 74: 7. 
3. Bhakuni DS and Joshi PP (1975). Alkaloids of Cocculus pendulus (forsk) Diels. Tetrahedron 31: 2575-2579.

4. Rabari H, Pandya S, Vidyasagar G \& Gajra B (2010). Pharmaconostic studies of Cocculus pendulus leaf. Inter J of Pharma and Bio Sci 1: 1-13.

5. Paul S, Saha D \& Chowdhury S (2012). Pharmacognostic studies on aerial parts of methanolic extract of Mimosa pudica. Asian J Pharm Tech 2: 101-103.

6. Saha D \& Paul S (2012). Pharmacognostic studies of aerial part of methanol extract of Borreria articularis. Inter $J$ of Pharma Innov 2: 67-71.

7. Parekh J, Jadeja D \& Chanda S (2005). Efficacy of aqueous and methanol extracts of some medicinal plants for potential Antibacterial Activity. Turk J Biol 29: 203-210.

8. Harnborne JB (1998). Phytochemical methods. $3^{\text {rd }}$ (eds.) Chapman and Hall; New York.

9. Huang X, Gao W, Zhao W, Zhang T \& Xu J (2010). Flavone and steroid chemical constituents from rhizome of Paris axialis. Zhongguo Zhong Yao Za Zhi 35: 29942998.

10. Barkatullah, Ibrar $\mathrm{M} \&$ Muhammad $N$ (2011). Evaluation of Zanthoxylum armatum $D C$ for in-vitro and in-vivo pharmacological screening. Afr J Pharm Pharmacol 5: 1718-1723.

11. Atta-ur-Rehman, Choudhary MI \& Thomsen WJ (2001). Bioassay Technique for Drug Development Harwood Academic Publishers 9-67.

12. Nasrin F (2013). Antioxidant and cytotoxic activities of Ageratum conyzoides stems. Inter Curr Pharma J2: 33-37.

13. Hosseinzadeh $\mathrm{H}$, Ramezani $\mathrm{M} \&$ Namjo $\mathrm{N}$ (2003). Muscle relaxant activity of Elaeagnus angustifolia L. fruit seeds in mice. J of Ethnopharmacol 84: 275-278.

14. Khan H, Saeed M, Gilani AH, Khan MA, Dar A \& Khan S (2010). The antinociceptive activity of Polygonatum verticillatum rhizomes in pain models. $J$ of Ethnopharmacol 127: 521-527.

15. Khan M, Khan AH, Khan S, Mahmood T, Khan PM \& Jabar A (2009). Antiinflammatory, analgesic and antipyretic activities of Physalis minima Linn. $J$ of Enz Inhib and Med Chem 24: 632-637.

16. Manohar D, Lakshman K, Shylaja H, Viswanatha GL, Rajesh S \& Nandakumar K (2009). Antidiarrheal activity of methanolic extracts of seeds of Lepidium sativum. J of Nat Remedies 9: 197-201.

17. Mathur R (2013). Phytochemical and antimicrobial evaluation of plant extracts of Enicostemma hyssopifolium. $J$ of Pharmacog and Phytochem 2: 30-36.

18. Barkatullah, Ibrar M, Nafees M, Rauf A \& Khan H (2015). Cytotoxic, acute toxicity and phytotoxic activity of Callicarpa macrophylla in various models. American $J$ of Biomed and Life Sci 3: 1-5.

19. Roslida AH, Erazuliana AK \& Zuraini Z (2008). Anti-inflammatory and antinociceptive activities of the ethanolic extract of Pluchea indica less leaf. Pharmacol 2: 349-360.

20. Morshed A, Hossain MH, Shakil S, Nahar K, Rahman S, Ferdausi D, Hossain T, Ahmad I, Chowdhury MH \& Rahmatullah $\mathrm{T}$ (2010). Evaluation of antinociceptive activity of two Bangladeshi medicinal plants, Kalanchoe pinnata (Lam.) Pers. and Lagerstroemia speciosa (L.). Pers Adv in Nat Appl Sci 4: 193-197.

21. Apu SA, Chowdhury FA, Khatun F, Jamaluddin AT, Pathan AH \& Pal A (2013). Phytochemical screening and Invitro evaluation of Pharmacological Activities of Aphanamixis polystachya (Wall) Parker Fruit Extracts. Tropical J of Pharma Res 12: 111-116.

22. Nesa NS, Titta AD, Piccapietra F, Dobias J, Nesatyy VJ, Suter MF \& Latmani RB (2015). Binding of Silver Nanoparticles to Bacterial Proteins Depends on Surface Modifications and Inhibits Enzymatic Activity. Environ Sci Technol 44: 21632168 . 
23. Sanmugapriya S, Oza G, Mewada A \& Sharon M (2015). Green synthesis of highly stable gold nanoparticles using Momordica charantia as nano fabricator. Schol Res Libr 4: 1135-1141.

24. Chandran SP, Chaudhary M, Pasricha R, Ahmad A \& Sastry S (2016). Synthesis of Gold

Nano triangles and Silver nanoparticles using Aloe Vera plant extract. Biotechnol Prog 22: 577-583.

25. Iqbal MJ, Hanif S, Mahmood Z, Anwar F \& Jamil A (2012). Antioxidant and antimicrobial activities of Amaranthus viridis leaf and seed extracts. J of Med Plants Res 6: 44504455.

26. Mattew CP, Patel AJ, Patel MA, Chauhan KR \& Solanki RP (2013). Pharmacognostical and physico-chemical standardization of leaves of Sida acuta. Asian J of Biochem and Pharma Res 1: 5761.

27. Irshad M, Aziz S, Rehman, H, Shahid M, Ahmed MN, Minhas FA \& Sherazi T (2012). Antioxidant and antimicrobial activities of essential oil of Skimmea laureola growing wild in the State of Jammu and Kashmir. $J$ of Med Plants Res 6: 1680-1684.

28. Meite S, Guessan JDN, Bahi C, Yapi HF, Djaman AJ \& Guina FG (2009). Antidiarrheal Activity of the Ethyl Acetate Extract of Morinda morindoides in Rats. Trop J Pharm Res 8: 201-207.

29. Sara S, Ibrar M, Barkatullah, Muhammad N \& Ehsan M (2013). Analgesic and gastrointestinal motility profile of essential oil from Myrtus communis leaves.

Phytopharmacol 4: 81-86.

30. Banga YY, Yang DJ, Chiu CH, Lin YL, Chen JW \& Chen YC (2015). Antioxidative and anti-inflammatory effects of polyphenol-rich litchi (Litchi chinensis Sonn.) flower water extract on livers of high fat diet fed hamsters. $J$ of Functional Foods 5: 44-52.
31. Janbaz G, Kaur M, Singh PS, Rahar S, Dhabliya F, Arya Y \& Shri R (2016). Pharmacognostic parameters of Eucalyptus globulus leaves. Phcog $J$ 4: 38-43.

32. Muhammad N, Ahmad M, Mehjabeen JN, Ahmad M \& Khan K (2012). Spasmogenic and spasmolytic activity of Gratiola officinalis Linn. J Biom Pharm Sci 1: 636-644.

33. Ahmad F, Hasan I, Chishti DK \& Ahmad H (2012). Antibacterial activity of Raphanus Sativus Linn. seed extract. Global J of Med Res 12: 26-34.

34. Adwan G, Abu-Shanab B \& Adwan K (2010). Antibacterial activities of some plant extract alone and in combination with different antimicrobials against multidrug-resistant Pseudomonas aeruginosa strains. Asian Pac J Trop Med 25: 266-269.

35. Peace ME, Chinweizu U, Ekaete EA \& Udeme E (2013). Antimicrobial activities of leaf and stem bark extracts of Blighia sapida. $J$ Plant Stud 2: 47-52.

36. Raza ST, Hussain S, Riaz H \& Mahmood $S$ (2013). Review of beneficial and remedial aspects of Cardiospermum halicacabum L. Afr J Pharm Pharmacol 7: 3026-3033.

37. Nisar M, Khan I, Simjee SU, Gilani AH, Obaidullah \& Perveen AH (2008). Anticonvulsant, analgesic and antipyretic activities of Taxus wallichiana Zucc. J Ethnopharm 116: 490-494.

38. Nesal ML, Munira M, Bristy AS, Islam MM, Chayan H \& Rashid HM (2015). Cytotoxic, anti-inflammatory, analgesic, CNS depressant, antidiarrheal activities of the methanolic extract of the Artocarpus lakoocha leaves. World J Pharm Sci 3: 167-174.

39. Wen LD, Jiang Y, Nagendra K, Prasad SL, Guoxiang J, He H, Mouming Z, Luo W \& Yang B (2014). Identification of Litchi leaf and evaluation of anticancer activity. $J$ Func Foods 6: 555-563. 